\title{
Mechanical Simulation and Analysis of The Construction Process of A large-span space latticed structure in Coal Yard
}

\author{
Xiaoli Yang ${ }^{1, ~ a, ~ J i a n g ~ Z h e n g ~}{ }^{2,3, b}$, Guangen Zhou ${ }^{3, c}$ and Bo Yang ${ }^{4, d}$ \\ ${ }^{1}$ Xi'an Eurasia University, Xi'an 710065, China \\ ${ }^{2}$ School of Civil Engineering, Xi'an University of Architecture \& Technology, Xi'an 710055, China \\ ${ }^{3}$ Postdoctoral Workstation, Zhejiang Southeast Space Frame Company, Hangzhou 311209, China \\ ayangxiaoli96@163.com, bzhengjiang94@163.com, '81347893@qq.com, '690247470@qq.com
}

Keywords: large-span; space latticed structure; construction mechanics; mechanical emulation; finite element analysis

Abstract: The structure of the coal yard is a four pyramid space latticed structure. In this paper, based on Midas/Gen finite element analysis software, the finite element model of the structure of the coal yard has been established, and the two methods which are one-time loading and considering the path effect are analyzed apart in the construction process. The analysis results show that the analysis method considering the path effect is quite different from that calculated by the one-time loading method. The former is closer to the actual state of the structure and makes the construction process safe and reliable. Through the above analysis, the safety and economy of the construction process can be guaranteed, and the internal force and deformation at the completion stage of the construction can meet the requirements of the design. It can also provide reference for the construction of similar structures.

\section{Introduction}

The traditional structural design theory is only used to analyze the effect of the complete stage of structure under different load cases and their combination, so as to ensure the safety and applicability of the building structure [1]. However, all buildings must go through a construction process and gradually forming structure, therefore in the process of the construction of the structure the stress and deformation mode are different with the design state [2]. In addition, according to incomplete statistics, more than $2 / 3$ of the collapse of the engineering structure occurred in the construction stage in China. [3]. Therefore, the mechanical simulation of construction process is very necessary, and one of its aims is to track the changes of the stress and deformation of the main structure and the temporary supporting structure during the construction process [4]. For example, in the engineering design state the structure is a complete spatial reticulated shell structures, but in the state of construction the structure is composed of discrete components gradually that will be assembled in the construction stage. Before completion of the construction, the structure is incomplete, cannot form a mechanical mode of space structure. So the stress mode during the construction process is completely different with the design state.

At present, the numerical methods of construction mechanics mainly include topology change method, time variant element method and finite element method [5-6]. In this paper, the finite element method is used in engineering which is good and easy to implement. The finite element method of the analysis of construction process is the basic method to simulate the whole process of mechanics. In this method the structure is divided into several steps by the actual construction scheme, and the stiffness and load of structure during construction process are activated respectively according to the time sequence of each construction step. The method considers the relationship between construction steps and cumulative effect and simulates the changes of the stress and deformation really of the whole construction process. Dividing the construction step is achieved through the activation and passivation of the structural elements. At first, the whole finite element structural model is established; and then according to the construction schedule the structural elements are activated and passivated gradually; and then the load and the corresponding boundary conditions of the construction step are applied, you 
can track the analysis of internal force and deformation of the development of changes in the structure of the construction process [1].

\section{General situation and structural features of this project}

Project overview. The steel structure of the coal yard is enclosed, and the four pyramid space shell structure is adopted, all of which are Q235B steel. The span of the cylindrical reticulated shell is $87.6 \mathrm{~m}$, the thinnest thickness is $2.3 \mathrm{~m}$; the length of the building is $422 \mathrm{~m}$, the length of the building is divided into four sections of temperature. There are $0.5 \mathrm{~m}$ deformation joints between each temperature section (refer with: Fig. 1), the length of each temperature section is about $105.5 \mathrm{~m}$, the top elevation of the structure is about $32 \mathrm{~m}$. Among them, the No. 1 zone and 4 zone are respectively equipped with gables. The design takes into consideration the coordination between the gable grid structure and the main grid structure, and the coordination has been given full consideration when making the construction plan.

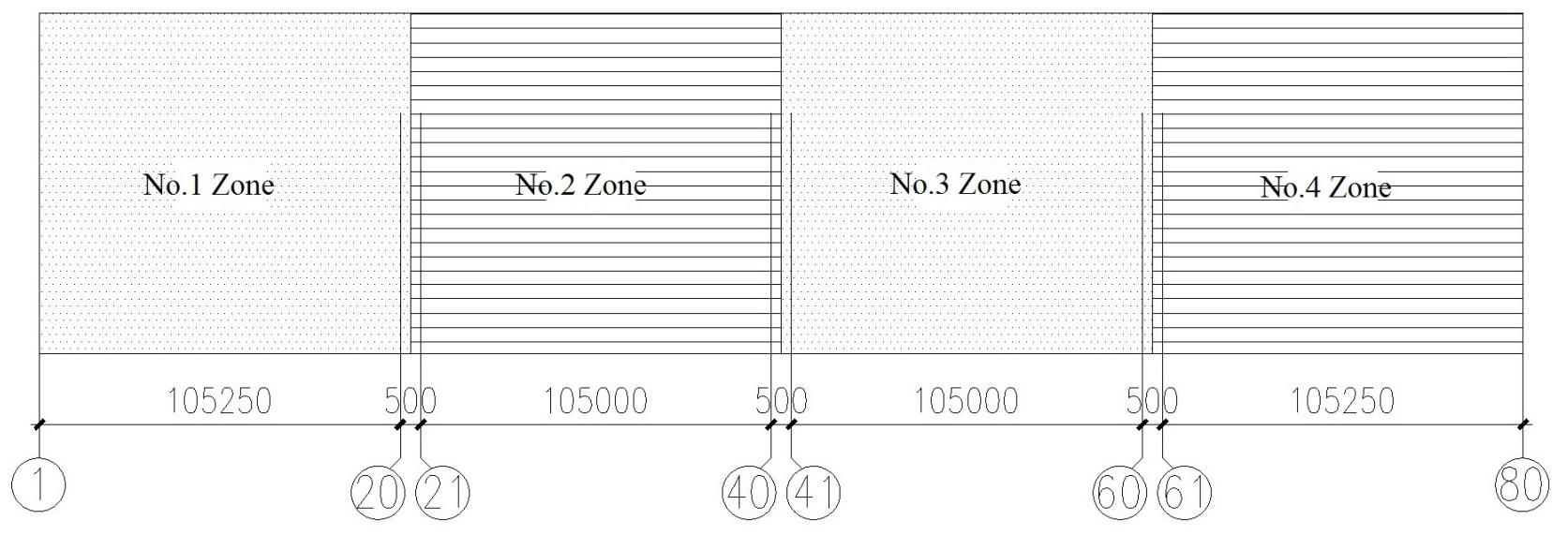

Fig.1 The structure zones of the coal yard

Structural features. The height and span are relatively large. The steel structure works with high span and span, weak rigidity, complex construction conditions, and dynamic structural internal force and deformation in the construction process, so it is necessary to make a systematic and detailed analysis.

The installation accuracy is difficult to control, and the requirements of construction quality are high. The steel structure is a reticulated shell structure, and it is difficult to control the development of internal force and deformation of the steel member under the influence of the dead weight and its additional bending moment. With the change of the weight of the structure, construction loads, it will bring uncertainties to the displacement of the main structure in the construction, at the same time, accurate measurement of the main structure will be impacted, the difficulty of the installation accuracy control of the structural components will increase. In the process of construction, it must take the necessary measures to adjust and eliminate the installation error, determine the control measures of measurement and the pre deformation. The deformation is controlled in the range of permission to ensure each structural component in the accurate position.

The construction work quantity is large and tight the construction period is tight. The construction period of the project is tight, the total weight of the steel structure is large, the number of the components is large, the model is complex, and the installation is difficult. Moreover, the construction site is relatively small, so that the steel structure installation components are difficult to pile up, and the pressure for the installation time of the structure is enormous. 


\section{Selection of construction plan}

Because the roof span and area of the project is large, the steel structure is adopted. The comprehensive installation method is adopted, which combines the basic unit lifting method with the cantilever installation method. A locally stable basic structure unit in the longitudinal central of the whole structure is selected to construct. This basic unit is divided into three parts. Two parts on the side are installed first and then the middle part is installed. In order to install the space steel structure, it is necessary to set a reasonable temporary support structure [7]. In this project every basic installation unit sets two temporary support frames (refer with: Fig. 2). The advantages of this method are that it can greatly reduce the use of scaffolding; the performance requirements of lifting equipment are not high; the installation and demolition period of temporary support frames is short, the construction is convenient, and it is less affected by the site conditions. The disadvantages are that there are many construction conditions, and the structural internal force and deformation of the construction process are in dynamic change, so it is necessary to make a systematic and detailed mechanics analysis.
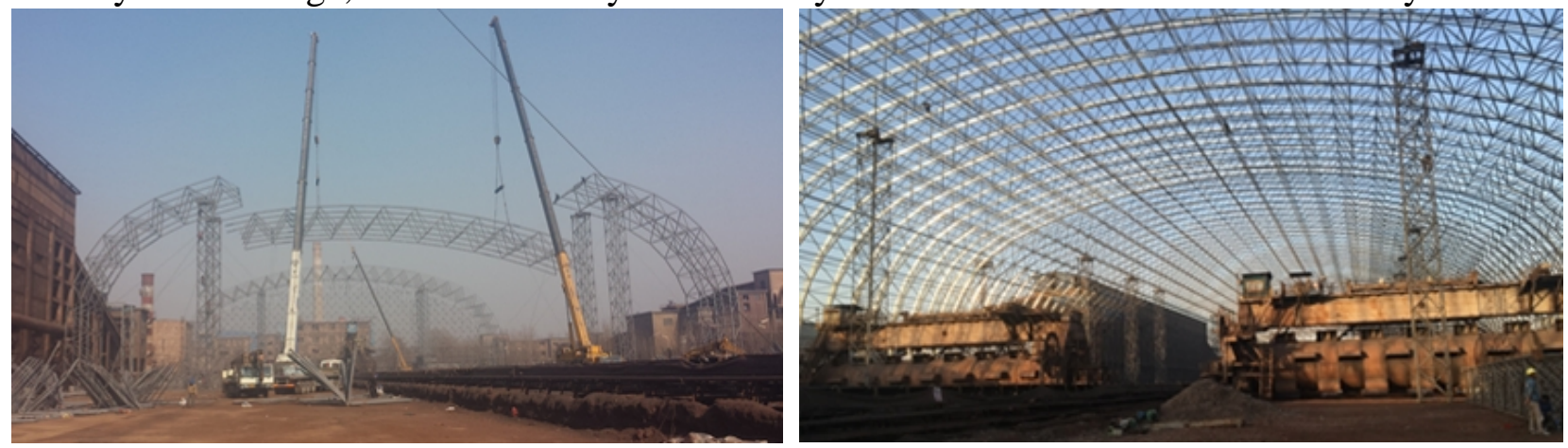

Fig. 2 The layout of the temporary support frames

The basic installation unit is determined according to the structural characteristics of the project and the actual situation at the scene. The installation basic unit of No.1 zone is arranged on the 2-4 axis (refer with: Fig. 3), the basic unit of No.2 is arranged on 34-36 axis, the basic unit of No.3 is arranged on 54-56 axis, the basic unit of No.4 is arranged on 77-79 axis. In No.1 and 4 zone, after the completion of the construction of the basic unit and the gable wall, the rest of the grid frame is installed by the cantilever installation method. The process of installing components between two columns is one construction step. After completion of this construction step, the temporary support frames in last construction step will be demolished to removed and installed in the next construction step. There are four temporary support frames. The four temporary support frames will be unloading at the same time when they are removed to the next construction step. As the length of the article is limited, only the analysis and calculation results of No.1 zone are enumerated.

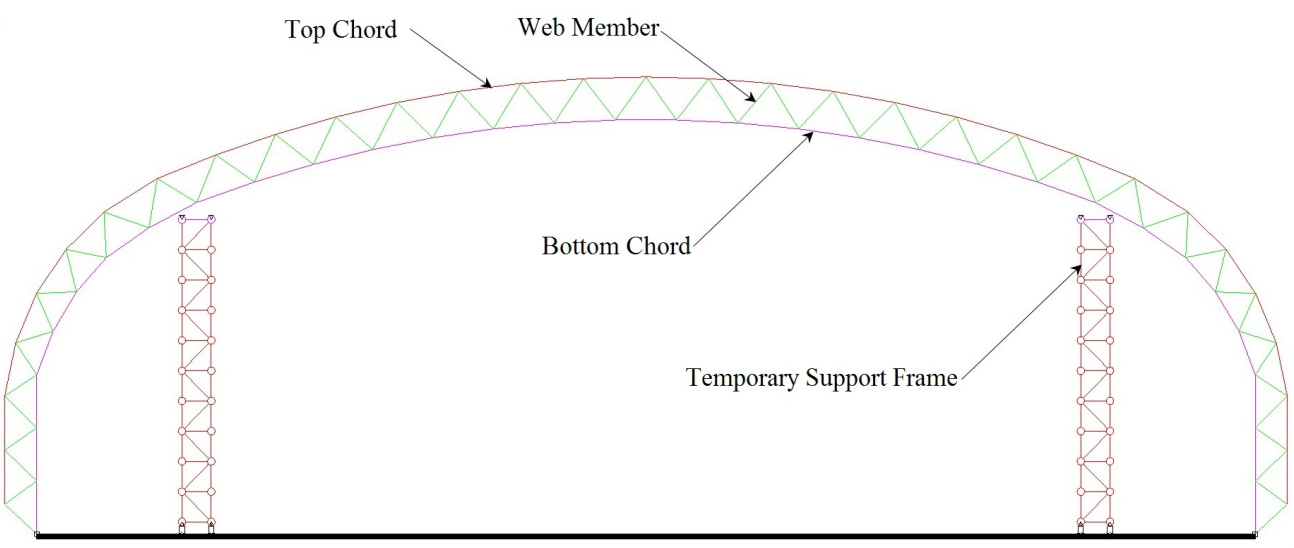

Fig.3 The installation basic unit 


\section{The method of mechanics' emulation}

In the structural analysis, the establishment of the finite element model is a key procedure, which directly affects the accuracy of the analysis results. The key to establish the model of structural analysis during construction is to make the model's rigidity, load and the construction steps used in the analysis accord with the actual situation as much as possible. This project uses finite element method for simulation, in order to ensure the accuracy of the calculation results, the model input material and size are the same as the actual structure, and the temporary support frames are simulated with an elastic support according to the really rigidity. The structure is welded by steel tube, and the beam element which can transfer the bending moment is used to simulate the main structural members.

Using finite element method to analyze the construction process of structure, according to the construction schedule, the progress of the construction phase will be divided into several construction steps. In the analysis of a construction step, the program will block all the components of the later construction stage and the load conditions and boundary conditions that should be loaded later, allowing only the components completed prior to the procedure to take part in the analysis. For example, in the first step of the calculation, all components after the completion of the first construction step are blocked, only activating the components in this step. The calculation process by considering the time dependence effect accumulation model is the way to carry on the analysis. The structural internal forces and deformation of each stage are obtained. In the next stage, the analysis will be adjusted to the model according to the new deformation, so that the dynamic process of construction can be simulated.

\section{Construction simulation analysis procedure}

The key to establish the structural analysis model of construction process is to make the calculation model accord with the reality as much as possible, so as to ensure the feasibility of the simulation. According to the construction sequence the whole finite elements are passivated at first. And then the finite elements will be activated gradually according to the construction schedule. In this process the boundary and load will be activated also. This analysis method not only satisfy the continuity of the construction process, also ensure the transmitting of deformation and internal force among the construction steps. Part of the model of construction steps are shown in the Fig. 4.
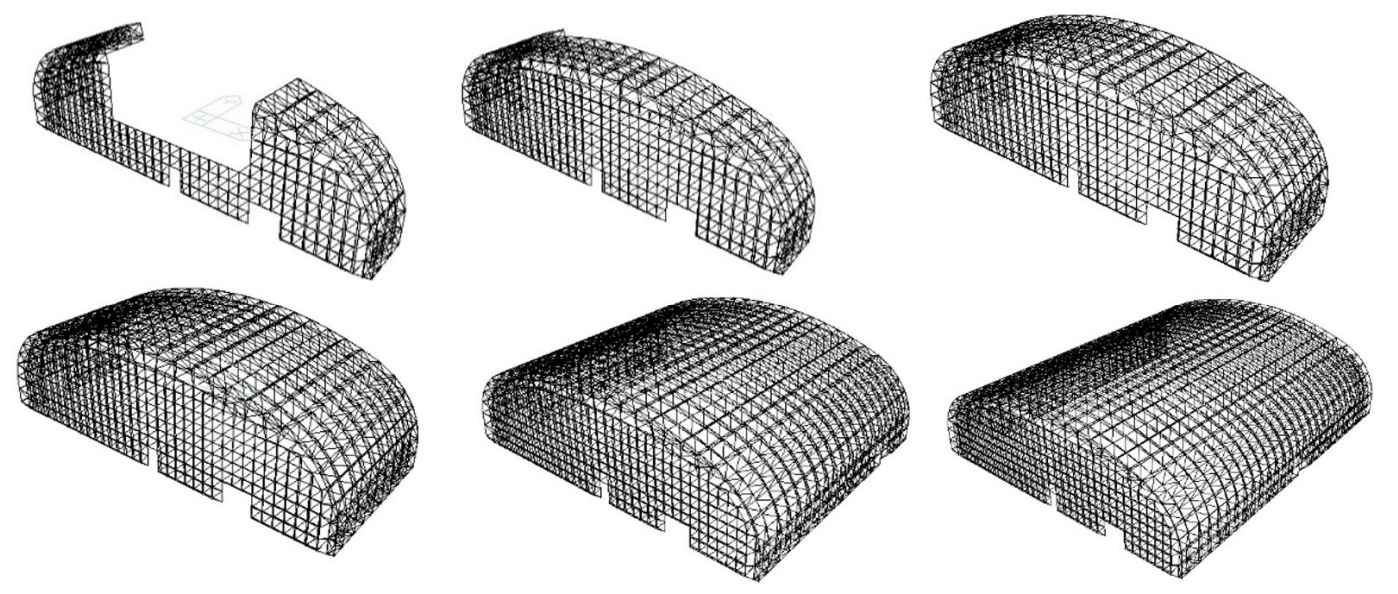

Fig.4 Models of partial construction stage

The construction sequence of the No.1 of this project is as follows:

(1) the main structure of a district is divided into 27 construction stages (refer with: Fig. 4) to simulate the installation of the main structural members;

(2) lifting of basic installation unit;

(3) lifting of gable wall;

(4) cantilever installation. 


\section{Finite element analysis of construction process}

The design analysis of the structure under the state of one-time loading (that is, in the design state, considering only the dead load) and the construction process analysis are made. In general, the designers analyze the structure on condition that the overall stiffness of the structure has been formed, not considering the effect of construction process. However, in the process of real construction, the components are gradually added. There must be a difference between the actual state of internal force and deformation and the design state when the construction is completed. Therefore, the choice of construction methods should be aimed at minimizing the difference between the two, ensuring the safety and use of the structure [8].

Fig. 5 gives the deformation and stress of the structure analysis under one-time loading; Fig. 6 gives the analysis results considering the effect of construction process. The maximum displacement and stress during the construction process are occurred in the same region. Because of the symmetry of the structure the half structure members (refer with: Fig. 7) of the No. 10 axis are adopted to show the analysis result. The comparison results of design state and construction state are shown in Fig. 8

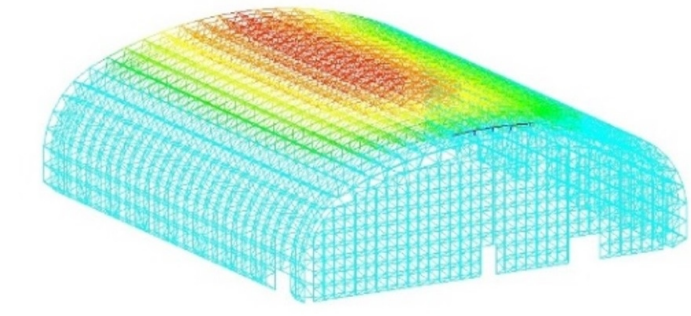

(a) the deformation of $\mathrm{DZ}(\mathrm{mm})$

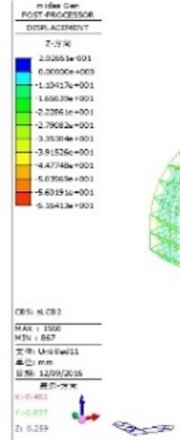

it

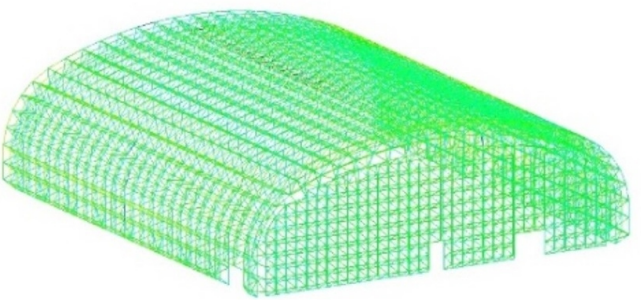

(b) the stress $\left(\mathrm{N} / \mathrm{mm}^{2}\right)$

Fig.5 The structure analysis under one-time loading

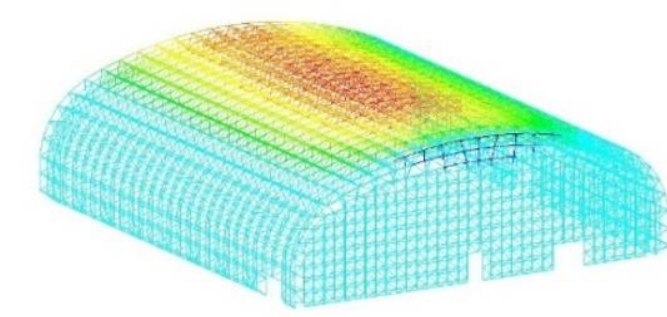

(a) the deformation of DZ (mm)

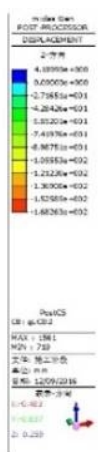

Fig.6 The structure analysis considering the effect of construction process

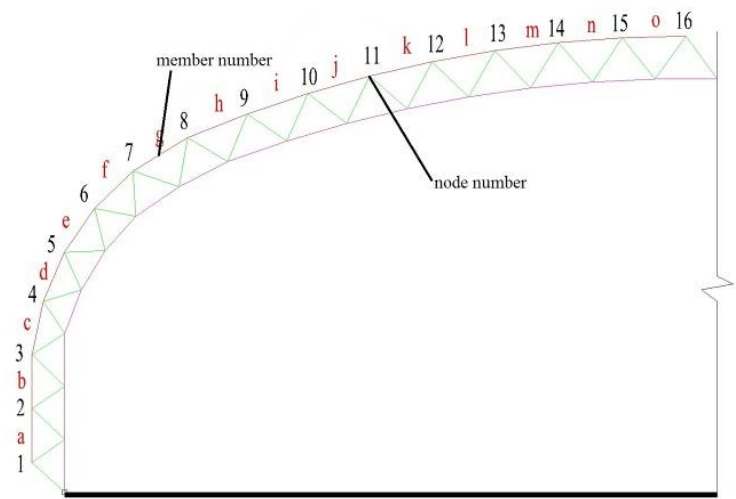

Fig.7 The half structure members of the No. 10 axis 


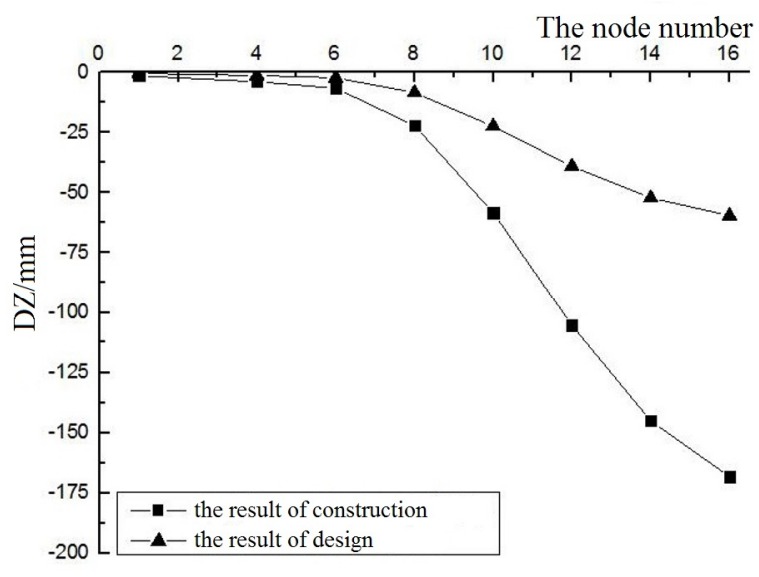

(a) the deformation of DZ (mm)

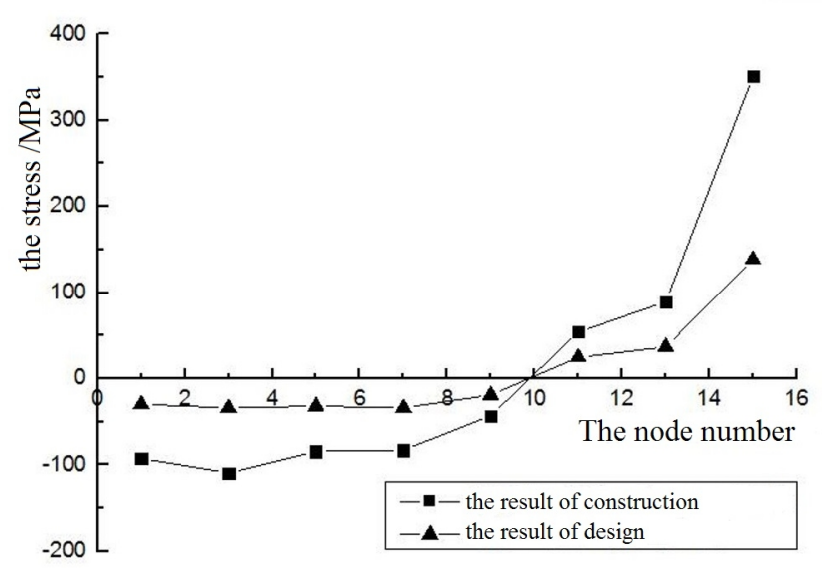

(b) the stress $\left(\mathrm{N} / \mathrm{mm}^{2}\right)$

Fig. 8 The comparison results of design state and construction state

From the above analysis, the structure during the construction period, the vertical deformation data show that the result of one-time loading analysis is obviously different with the result considering the effect of construction process. It is necessary to consider the influence of the construction process. As shown in Fig. 8, the two simulation methods have the same deformation trend, indicating that the construction method is reasonable. Secondly, from the simulation results, some members have the large deformation, the pre-deformation value must be applied in these members to reduce the deformation. The stress of part of the members is large, and these members must be changed to ensure the safe of the structure.

During the construction and installation of the structure, the configuration of the installed members is constantly changing under the construction load of the new installation members [9]. Therefore, in the installation of the gable wall, because the basic unit of the top is not closed, during construction the outer deformation of the gable wall must be paid attention to controlling.

\section{Conclusion}

In this paper, the construction process of a large-span steel structure is simulated and analyzed, and the following conclusions are drawn:

(1) through the simulation analysis of the whole construction process of a large-span steel structure of a coal yard, the mechanical and deformation performance of the structure under the actual construction scheme can be obtained, which provides a reference for the actual construction.

(2) for the construction of large span structure, it is necessary to consider the influence of the construction process. At the same time, the corresponding measures should be taken in advance, such as the displacement pre-deformation of the members with larger displacement, and the replacement of some of the members according to the analysis results. Under the analysis of the two methods, although the deformation and internal forces are different with the design state, but the overall trend is consistent. Through pre-deformation and the replacement of part of the members, could meet the design requirements.

(3) by the comprehensive installation method which combines the basic unit lifting method with the cantilever installation method is reasonable. By this construction method no large lifting equipment is needed during the construction process, the scaffolding has less area and low cost, the cost of construction is obviously reduced. At the same time, it also solves the problem of the installation of the latticed shell under the condition that the scaffold is unable to set up, and provides a reference for the construction of this kind of structure. 


\section{Acknowledgements}

This work was financially supported by the Cooperative Innovation Program of Shaanxi Province (2016XT-12).

\section{References}

[1] Liu Xuewu, Guo Yanlin, Zhang Qinglin, et al. Analysis of structural internal force and deformation during construction of CCTV new station main building [J]. Industrial Building, 2007, 37 (9): $22-29$.

[2], Zheng Jiang, Hao Jiping, Wang Yu, Lining, et al. Mechanical simulation of unloading process of large span steel roof. [J]. Architectural Science, 2014,30 (3): 80-84.

[3], Zhao Guofan, Gong Jinxin. Reliability of life cycle of engineering structures [M]. Beijing: China Railway Publishing House, 2004.

[4] Hao Jiping, Zheng Jiang, Wang Xiantie, Yang Xiaoli. Mechanical simulation and analysis of the construction process of the main stadium of the Universiade [J]. Journal of Xi'an University Of Architecture And Technology (Natural Science Edition), 2010,42 (4): 458-463.

[5] Cao Zhiyuan. Construction mechanics and time change mechanics foundation for civil engineering analysis [J]. Proceedings of the Civil Engineering Society, 2001,34 (3): 41-46.

[6] Cui Xiaoqiang, Guo Yanlin, Ye Keming. Analysis of structure of large span steel structure construction method of [J]. Engineering Mechanics, 2006,23 (5): 83-88.

[7], Guo Yanlin, Cui Xiaoqiang. Some technical problems during construction of large span complex steel structures [J]. Industrial Architecture, 2004,34 (12): 1-5.

[8] Guo Xiaokang, Li Peng. Analysis of [J]. construction technology of whole process simulation of coal construction of Zhanjiang iron and steel base type B, 2015,44 (20): 86-89.

[9] Zheng Jiang, Ge Hongpeng, Wang Xiantie, He Zhen, Louyong. Local configuration constraint death element method and its application in construction mechanical analysis [J]. Journal of building structures, 2014,33 (8): 101-108. 